\title{
Emerging Putative Biomarkers: The Role of Alpha 2 and 6 Integrins in Susceptibility, Treatment, and Prognosis
}

\author{
James R. Marthick and Joanne L. Dickinson \\ Menzies Research Institute Tasmania, University of Tasmania, 17 Liverpool Street Hobart, TAS 7000, Australia \\ Correspondence should be addressed to Joanne L. Dickinson, jo.dickinson@utas.edu.au
}

Received 10 February 2012; Accepted 17 May 2012

Academic Editor: William Grizzle

Copyright ( $) 2012$ J. R. Marthick and J. L. Dickinson. This is an open access article distributed under the Creative Commons Attribution License, which permits unrestricted use, distribution, and reproduction in any medium, provided the original work is properly cited.

\begin{abstract}
The genetic architecture underpinning prostate cancer is complex, polygenic and despite recent significant advances many questions remain. Advances in genetic technologies have greatly improved our ability to identify genetic variants associated with complex disease including prostate cancer. Genome-wide association studies (GWASs) and microarray gene expression studies have identified genetic associations with prostate cancer susceptibility and tumour development. The integrins feature prominently in both studies examining the underlying genetic susceptibility and mechanisms driving prostate tumour development. Integrins are cell adhesion molecules involved in extracellular and intracellular signalling and are imperative for tumour development, migration, and angiogenesis. Although several integrins have been implicated in tumour development, the roles of integrin $\alpha_{2}$ and integrin $\alpha_{6}$ are the focus of this paper as evidence is now emerging that these integrins are implicit in prostate cancer susceptibility, cancer stem cell biology, angiogenesis, cell migration, and metastases to bone and represent potential biomarkers and therapeutic targets. There currently exists an urgent need to develop tools that differentiate indolent from aggressive prostate cancers and predict how patients will respond to treatment. This paper outlines the evidence supporting the use of $\alpha_{2}$ and $\alpha_{6}$ integrins in clinical applications for tailored patient treatment.
\end{abstract}

\section{Introduction}

The diagnosis of aggressive prostate cancer at an early stage is crucial for successful management; however, clinicians still lack the diagnostic tools to identify indolent tumours from those likely to be aggressive and with a propensity to metastasise. Currently, the prostate-specific antigen (PSA) test still remains a widely used marker of choice for diagnosis and monitoring the progression of prostate cancer [1]. Thus molecular markers that can characterise individuals with a genetic susceptibility to prostate cancer identify malignant potential, provide real-time tumour surveillance, and potentially offer therapeutic intervention represent a major focus of current research. The advent of highthroughput genetic mapping technologies has not only highlighted the heterogeneity and complexity of the disease but also identified key molecules driving prostate cancer development and progression, and these molecules include the integrins.
The integrins represent a large family of cell surface receptors that are responsible for cellular adhesion and complex formation with ligands within the extracellular matrix (ECM). They are noncovalent, heterodimeric, transmembrane compounds, which extend from within the cytoplasm, spanning the cellular membrane, into the ECM [2-4]. The structure of these integrins is highly conserved between higher and lower organisms and is thought to highlight their importance to multicellular organisms [5]. To date, 18 alpha and 8 beta subunits have been identified, which form 24 heterodimers. These subunits can, in some cases, be further subdivided into "variants" created by alternate mRNA splicing events [6].

Individual integrins can form multiple heterodimers; for instance, $\beta_{1}$ can form 12 separate complexes. Most, however, form only one or two [2]. The collagen-binding integrin $\alpha_{2}$ binds only with $\beta_{1}$ and the laminin-binding $\alpha_{6}$ only with $\beta_{1}$ and $\beta_{4}$. Many integrins can be considered promiscuous as they can have multiple ligands; for example, 
tenascin, collagen, and laminin are all ligands for $\alpha_{2}$. Integrin promiscuity perhaps reflects the need to initiate different cellular processes using the same available ECM proteins [2]. For example, the ligand fibronectin binds to both $\alpha_{5} \beta_{6}$ and $\alpha_{5} \beta_{1}$, where $\alpha_{5} \beta_{6}$ stimulates cell migration and $\alpha_{5} \beta_{1}$ suppresses it $[7,8]$. The ability of integrins to bind to multiple ligands is thought to be an advantage when the response is more important than the ECM protein signalling it, for example, in wound healing [9].

Integrins are key mediators in a number of cellular processes including cell survival, proliferation, cell migration, angiogenesis, and lymphangiogenesis [10]. Cell migration and motility are crucial to maintain and promote healthy cell development, wound healing and immunity. They are complex processes requiring tightly regulated and coordinated intracellular signal transduction with the ECM. The aberrant expression of key molecules, such as the integrins required for cell-to-cell and cell-to-matrix interactions, is hypothesised to lead pathogenic phenotypes, such as neoplasia and progression towards the metastatic phenotype [11]. Thus as modulators of cell adhesion, the integrins are now thought to play a key role in prostate cancer tumourigenesis. Furthermore, several of these integrins represent excellent therapeutic targets. Inhibitors targeting selected integrins have already reached Phase II and Phase III human trials for glioblastoma, lung, and breast cancers [12]. In addition, work on therapeutics targeting the $\alpha_{2}, \alpha_{6}$, and $\beta_{4}$ subunits has commenced, with several of these currently undergoing validation in human trials.

The majority of deaths associated with prostate cancer are the direct result of metastatic disease, which is thought to arise as tumour cells escape via the basement membrane, through the prostate capsule, where they are transported throughout the body. These cells are then able to disseminate and propagate secondary lesions [13]. Integrins provide the traction for tumour cell invasion by interacting with the extracellular matrix [14]. The invasion process arises via the breakage of actin filaments at the leading edge of the cell migratory path, which leads to dissociation between the cell and the extracellular matrix proteins [15], pointing to a critical role for integrins in this process. Over $80 \%$ of prostate cancer deaths incur metastases to bone [16]. The $\alpha_{2}$ and $\alpha_{6}$ integrins are the primary ligands for collagen and laminin, which are found abundantly in bone, and these integrins therefore are of interest in prostate tumour progression.

The development of the pathogenic phenotype is thought to arise via $\alpha_{2}$ integrin eliciting the decay of ECM proteins, which is regulated by matrix metalloproteinases (MMPs). Ivaska and Heino [2] outline an interesting hypothesis by which MMP-1 and -2 mediated by integrins are able to degrade collagen. According to their hypothesis $\alpha_{2} \beta_{1}$ integrin binding to the fibrillar collagen matrix elicits the production of MMP-1, which degrades the collagen to gelatin, exposing an aspartate-glycine-arginine (RGD) site. The RGD site is then recognised and bound by $\alpha_{\mathrm{v}} \beta_{3}$ which results in the upregulation of MMP-2 further degrading the gelatin matrix thus completing the process [2]. Supporting this theory is that cells expressing collagen-binding integrins $\alpha_{2} \beta_{1}$ and $\alpha_{2} \beta_{2}$ display marked changes in expression in response to collagen [17], thus highlighting the intrinsic relationship between these integrins and ECM ligands.

\section{Gene Expression in Tumours}

Expression of $\alpha_{2}$ and $\alpha_{6}$ integrin genes appears to be integral in prostate cancer progression. Studies of hormone refractory prostate cancer clearly demonstrate that $\alpha_{2} \beta_{1}$ has a major role in prostate cancer cells adhering to the bone matrix $[18,19]$. The ability of $\alpha_{2} \beta_{1}$ to signal and interact with collagen in the bone microenvironment [20] may be particularly pertinent to the progression of prostate cancer. In normal prostate tissue, expression of $\alpha_{2} \beta_{1}$ is restricted to basal epithelial cells. During the normal differentiation of basal cells to intermediate cells, there is loss of substratum adhesion with an associated decrease in expression of integrins including $\alpha_{2} \beta_{1}[21]$.

Prostate basal epithelial cells also express the $\alpha_{6}$ subunit. Studies of $\alpha_{6}$ expression have reported that during progression from prostatic intraepithelial neoplasia to prostate carcinoma, $\alpha_{6}$ expression is maintained [22, 23], although $\alpha_{6} \beta_{4}$ expression diminishes and there is an increase in $\alpha_{6} \beta_{1}$. This increased expression is possibly due to a loss of $\beta_{4}$ expression associated with differentiation. It is also known that, in prostate tumour development, the $\alpha_{6}$ subunit can be cleaved to a truncated form, $\alpha_{6 \mathrm{p}}$, which is no longer able to interact with the substratum [22]. Examinations of $\alpha_{2} \beta_{1}$ expression at different stages of prostate tumour development have concluded that $\alpha_{2}$ is initially downregulated in precursor lesions, leading to increased metastatic behaviour; however, variable aberrant expression patterns are observed [18, 21, 24-26].

Compelling evidence for $\alpha_{2} \beta_{1}$ as a modulator of tumour initiation and progression in prostate and breast cancer has recently been provided by Ramirez and colleagues [12]. A transgenic mouse model was employed to examine the role of $\alpha_{2} \beta_{1}$ in breast cancer, generating an $\alpha_{2} \beta_{1}$-null and MMTV-c-erbB2/Neu (mouse mammary carcinoma model) oncogene transgenic mouse cross. A significantly increased number of tumours and metastatic lesions were found in $\alpha_{2} \beta_{1}$-null cross mice, with an observed increased rate of tumour intravasation and increased numbers of circulating tumour cells compared with $\alpha_{2} \beta_{1}$-wild-type crosses. Ramirez and colleagues [12] also accessed public microarray data to validate their findings in human studies. Significantly reduced expression of $\alpha_{2}$ in breast carcinoma compared with normal breast tissue was demonstrated. Furthermore, it was revealed that reduced $\alpha_{2}$ expression correlated highly with the presence of metastatic lesions, poor prognosis, and overall reduced survival in breast cancer sufferers. Examination of the $\beta_{1}, \alpha_{3}$, and $\alpha_{1}$ integrins showed no such associations [12]. A similar trend was observed in prostate tumour development with a progressive downregulation of $\alpha_{2}$ expressions associated with prostate tumour progression from the precursor lesion prostatic intraepithelial neoplasia (PIN), to carcinoma of the prostate and then to metastatic tumours.

A notable and repeated finding is that $\alpha_{2}$ is highly expressed by a subpopulation of cells in prostate tumours. 
Studies aiming to isolate subpopulations of tumour cells with "stem cell-like" properties have found that these "sidepopulations" express high levels of $\alpha_{2} \beta_{1}$ integrin [27]. In addition, selected subpopulations of the PC-3 prostate cancer cell line, with stem cell-like activity and the ability to initiate serially transplantable tumours are also characterised by expression of high levels of the $\alpha_{2} \beta_{1}$ integrin [28]. Evidence suggests that these "cancer stem cells" (CSCs) are a subpopulation of $\mathrm{CD} 44^{+}$cells found within solid tumours [29]. Guzmán-Ramírez and colleagues [30] have also isolated cells from resected human prostate tumour tissue. Serial passaging of these tumour-derived cell populations was termed "prostaspheres" and resulted in a subpopulation with "stem cell like properties such as self-renewal and high clonogenic potential"; these cells were found to express high levels of both the $\alpha_{2}$ and $\alpha_{6}$ integrins. Additionally, in murine models, CSCs been shown to mediate EMT [29]. Prostate tumour stem cells displaying high levels of $\alpha_{2} \beta_{1}$ expression have also been described by Collins et al. [31] and Miki et al. [32]. These results suggest that there exists a subpopulation of tumour cells with dysregulated $\alpha_{2}$ and $\alpha_{6}$ integrin expression that perpetuate progression and tumour metastasis.

\section{Integrins $\alpha_{2}, \alpha_{6}$ and the Genetics of Prostate Cancer}

Age, race, and family history of prostate cancer remain the strongest risk factors for developing this disease. Recent advances in whole-genome analysis technologies have permitted the advent of high-density single nucleotide polymorphism (SNP) arrays for mapping and the use of genome-wide association studies (GWASs) to examine the underlying genetic contributors to prostate cancer. Since 2008, more than 15 GWASs have targeted prostate cancer, identifying more common susceptibility variants than that for any other cancer. A summary of the 46 GWAS variants thus far identified and their location is outlined by Goh et al. [33]. Whilst GWAS have to date identified more than 40 disease susceptibility variants, these 40 variants only explain approximately $30 \%$ of an individual's heritable risk of developing prostate cancer [34]. Thus a significant proportion of the genetic contributors to prostate cancer remains to be discovered. The phenomenon of "missing heritability" is not only relevant to prostate cancer, but is the current focus of genetic research in many complex diseases as well. Furthermore, GWAS are identifying common variants and these are generally of small to moderate effect size. This is true for most GWAS of complex disease; of the hundreds of variants discovered by GWAS, more than $80 \%$ lie outside of coding regions, and thus their functionality remains to be determined [35].

Attempts have been made to utilise known risk variants to develop polygenic risk calculators for prostate cancer with limited success, even when presence of family history is included in the model [36]. Zheng and colleagues [37] combined 16 known risk SNPs in five chromosomal regions and identified that, when combined with a family history, they were estimated to account for $46 \%$ of prostate cancer burden in a large Swedish cohort. More recently, Aly and colleagues [36] developed a logistic regression model based upon 35 GWAS identified variants, to determine whether a prostate biopsy should be collected for diagnosis. The model was able to reduce the number of biopsies required by $22.7 \%$ but was not able to distinguish between aggressive and nonaggressive prostate cancer [36]. In addition, Pashayan and coauthors [38] utilised 31 GWAS identified SNPs in a polygenic model and was able to reduce the number of men screened by $16 \%$, but with a concomitant loss of sensitivity (3\% increase in missed diagnoses). However, the model was able to identify younger men at increased risk. Our inability to identify the genetic risk factors explaining the majority of heritable risk is thus impeding our ability to develop accurate diagnostic tools to assist clinicians in the identification of clinically significant disease.

It is thought that "missing heritability" may be explained, at least in part, by rare variants. Rare mutations in the BRCA2 gene are already known to contribute to a small but significant proportion of prostate cancers with earlyonset disease and poor survival $[39,40]$. There has been a recent call to apply new genetic analysis technologies such as next-generation sequencing to familial studies as a powerful alternative approach for discovering the rarer variants likely to be of greater effect size associated with complex disease risk [41]. This approach has recently proven successful for several complex diseases including multiple sclerosis [42] and prostate cancer. Next-generation sequencing of a previously identified region of linkage on chromosome 17q21-22 has identified rare mutations in the HOXB13 gene associated with disease in familial prostate cancer [43].

Epigenetic alterations may also contribute to "missing heritability" associated with disease. Epigenetic phenomena such as heritable changes in DNA methylation, histone modifications, and chromatin remodelling can alter gene expression. Methylation at cytosine residues in CpG islands is well documented as influencing gene expression in numerous cancers [44], including prostate cancer $[45,46]$. DNA methylation can aberrantly affect gene transcription by either preventing the transcriptional machinery from binding to the target region or by facilitating an interaction with chromatin remodelling proteins [47]. Unsurprisingly, the nature of these interactions is highly complex. Both global hypomethylation $[48,49]$ and hypermethylation of gene promoters are known to occur in prostate tumour development [50]. The methylation status of gene promoters has already been identified as predicative biomarkers with important clinical applications, for example, GSTP1 [51]. Whilst improved understanding of the role of heritable epigenetic modifications in prostate tumours is needed, the reversibility of epigenetic modifications and their propensity to arise before somatic mutations make them attractive targets for biomarkers and therapeutic intervention [47].

Most recently, emphasis has been placed on our inability to distinguish those aggressive tumours with a propensity to metastasise from more indolent disease that results in the unnecessary treatment of many men whose disease may never progress to clinical relevance. Given that current 
prostate cancer treatments such as prostatectomy, chemical castration, and radiation therapy are associated with considerable morbidities including impotence, incontinence, infection, and death, we urgently require a better understanding of the underlying drivers of this disease. The application of molecular screening techniques that can elucidate this subset of aggressive disease is therefore a focus of current translational research. Attempts to characterise SNPs associated with aggressive disease have produced conflicting results. Variants at $8 \mathrm{q} 24$ have been reported to be associated with increased risk of developing aggressive disease in a cohort of 823 Caucasian French men [52]. Furthermore, Helfand and colleagues [53] have also shown that the microsatellite loci (DG8S737) on 8q24 are significantly associated with aggressive disease $(P=0.04)$. Carriers of DG8S737 were significantly more likely to have a Gleason score greater than 7 and lymph node metastases [53]. FitzGerald and colleagues [54] also report that a variant on $15 \mathrm{q} 13$ (rs6497287) is significantly associated with aggressive prostate cancer $(P=$ 0.004). While these studies represent important advances in our ability to screen for aggressive prostate cancer, they are currently unreplicated; therefore, the ability of these risk SNPs to accurately predict disease aggressiveness remains to be determined [33]. Issues also remain as to whether GWAS-identified genes are true cancer susceptibility genes or rather, those associated with tumour progression, as tumour detection requires a minimum size before it can be discovered [55]. We are yet to identify many of the true causal variants underlying the identified GWAS-identified SNP associations. Our lack of understanding of how these genetic associations drive prostate tumour development currently hampers translation of many of these genetic findings into the clinic.

Genetic studies have previously identified polymorphisms residing within genes coding for the $\alpha_{2}$ and $\alpha_{6}$ subunits as significantly associated with disease, and gene expression profiling studies of prostate tumours have established altered gene expression in prostate tumours. For example, the GWAS conducted by PRACTICAL utilising over 30,000 cases and controls identified the variant rs12621278 in ITGA6 as significantly associated with prostate cancer susceptibility (allelic odds ratio $=0.75$, confidence interval $=$ $\left.95 \% P=8.7 \times 10^{-23}\right)$ [56]. Interestingly, this variant has also been highlighted as playing an important role in prostate cancer progression. Cheng and colleagues [57] screened 26 SNPs previously identified by GWAS, in 788 patients that had undergone radical prostatectomies to test for an association with aggressive prostate cancer. Of these 26 SNPs, five were associated with aggressive prostate cancer progression, the strongest of which was rs12621278. The risk allele of this SNP increased the risk of prostate cancer progression by 2.4fold $(P=0.0003)$ [57]. Genetic variants associated with prostate cancer have also been found in the ITGA2 gene which codes for the $\alpha_{2}$ integrin subunit. Utilising a familial linkage approach, FitzGerald and coauthors [58] identified a region on $5 \mathrm{p} 13 \mathrm{q} 12$ in a large pedigree, with multiple cases of prostate cancer. Subsequently, two SNPs were identified, one in the $3^{\prime}$-UTR (rs3212649) and another synonymous mutation in exon seven $(\mathrm{C} 807 \mathrm{~T})$, which increased prostate cancer risk both in the familial $(\mathrm{OR}=2.16, \mathrm{CI}=1.19$ $3.92)$ and combined datasets $(\mathrm{OR}=1.52, \mathrm{CI}=1.01-2.28)$. In addition, the C807T mutation has also been associated with an increased risk of developing oral and advanced breast cancers $[59,60]$. There is also mounting evidence that silent mutations in coding regions are not as silent as once thought and are able to alter the rate at which protein translation occurs [61] or interfere with splicing events and RNA structure [62]. Whilst the C807T mutation does not change the amino acid sequence, it has been associated with changes in expression of the $\alpha_{2}$ receptor on the cell surface [63].

The integrins also feature prominently in microarray studies of prostate cancer tissue. Combining the results with GWAS-identified variants with observed changes in gene expression can provide insight into the functional significance of these variants. Gorlov and colleagues [55] performed a meta-analysis on gene expression data derived from normal and tumour tissue and combined this with GWAS data to identify overrepresented gene families. Genes coding for the $\alpha_{2}, \alpha_{6}$, and $\beta_{4}$ integrins featured conspicuously amongst the most differentially expressed and most significantly associated with prostate cancer [55]. While the function of these variants remains to be determined, there is a large body of evidence supporting a role for these integrins in prostate tumour biology and therefore these integrins represent potential target candidate genes for use in diagnosis and prognosis. It is by gaining a thorough understanding of the genetic drivers of disease and their contribution to tumour biology that will permit effective translation of genetic findings in the clinical setting.

\section{Integrin $\alpha_{2} \beta_{1}$ as a Biomarker}

The accumulated experimental evidence outlined above suggests that monitoring integrins, in particular $\alpha_{2} \beta_{1}$, may be useful in tracking prostate cancer development. The dual nature of cell surface receptor and differential expression in prostate cancer has contributed to the development of new imaging tools for tracking and identifying prostate cancer cells in vivo. Molecular imaging provides real-time data in a noninvasive and sensitive manner [64]. Optical imaging probes targeting the Asp-Gly-Glu-Ala (DGEA) motif in integrin $\alpha_{2} \beta_{1}$ with near-infrared-fluorescent (NIRF) imaging have been developed by Huang and colleagues [65]. A Cy5.5conjugated DGEA peptide demonstrated high specificity in athymic mouse models with human PC-3 xenografts [65]. Crucially, the DGEA peptide worked exceptionally well as a ligand for $\alpha_{2} \beta_{1}$. However, the lack of quantitative data and challenges associated with optical imaging of deep tissues such as the prostate directed Huang and colleagues [65] to examine $\alpha_{2} \beta_{1}$ with nuclear imaging techniques.

Positron emission tomography (PET) scanning is currently the most common method for cancer diagnosis; however, problems arise in prostate cancer imaging using traditional methods such as (18)F-fluorodeoxyglucose (FDG) potentially due to the often slow growing nature of the disease [66]. Utilising a (64)Cu-labelled $\alpha_{2} \beta_{1}$ probe and the 
previously developed DGEA peptide conjugated with a bifunctional chelator, the integrin $\alpha_{2} \beta_{1}$ probes were used in a PC-3 mouse xenograft model with some success. Tumours showed significant probe uptake with high specificity [66] and have since been validated in PC-3, CWR-22, and LNCaP prostate cancer cell lines [67]. Integrin-targeted nuclear imaging focusing on $\alpha_{2} \beta_{1}$ represents a significant advance in molecular imaging of prostate cancer. These advances potentially provide an excellent method by which prostate cancer progression may be better assessed in the clinical setting. Furthermore, imaging techniques such as this may be able to distinguish between different tumour subtypes in a heterogeneous disease, thus facilitating an ability to provide tailored therapies utilising anti- $\alpha_{2} \beta_{1}$ integrin therapies.

\section{Integrin $\alpha_{2} \beta_{1}$, Diet, and Prostate Cancer}

Numerous epidemiological studies have sought to untangle the dietary components associated with risk of developing prostate cancer, and several of these have implicated lycopene as a potential protective agent these (reviewed by Giovannucci) [68], although some uncertainty remains [69, 70]. Lycopene is a powerful antioxidant, derived primarily from tomato-based products [71]. Intriguingly, malignant prostate cancer cell lines 22Rv-1, PC-3, and LNCaP display significantly reduced levels of $\alpha_{2} \beta_{1}$ when treated with lycopene [72]. In the same study, Buryeko and colleagues [72] also demonstrated that fish oil reduced $\alpha_{2} \beta_{1}$ expression in the more invasive cell lines LNCaP and PC-3. While the addition of lycopene and fish oil to cultured cell lines was able to reduce the expression of $\alpha_{2} \beta_{1}$, it was not able to attenuate the growth of the prostate cancer cell lines, suggesting that lycopene has an inhibitory role on migration and prostate cancer progression [72]. However, the use of lycopene and fish oils and chemopreventative agents remains controversial. Recently, clinical trials examining the efficacy of lycopene and fish oil in 84 men with low-risk prostate cancer use cDNA arrays after three months exposure [73]. However, no significant gene expression changes associated with taking lycopene or fish oil were identified [73].

\section{Therapeutic Targets and Angiogenesis}

The development of new blood vessels from preexisting ones requires interaction with the ECM [74]. Therefore, integrins can be viewed as a primary target for the prevention of angiogenesis associated with tumour progression. The role of integrins in tumour angiogenesis has been well described, with $\alpha_{v} \beta_{3}, \alpha_{5} \beta_{5}, \alpha_{5} \beta_{1}, \alpha_{4} \beta_{1}, \alpha_{6} \beta_{1}, \alpha_{9} \beta_{1}, \alpha_{6} \beta_{4}, \alpha_{6} \beta_{1}, \alpha_{2} \beta_{1}$, and $\alpha_{1} \beta_{1}$ all implicated in its pathology $[14,75]$. The $\alpha_{\mathrm{v}}$ and $\alpha_{5}$ integrins have been the focus of much of this research; however, the $\alpha_{2}$ integrins have also shown great promise as a target for antiangiogenic agents.

Evidence suggests that angiogenesis is mediated by the vascular endothelial growth factor receptor pathway 1 (VEGFR1) and integrin $\alpha_{2} \beta_{1}$ [75]. Furthermore, $\alpha_{2} \beta_{1}$ is thought to be proangiogenic, with abrogation of the $\alpha_{2} \beta_{1}$ gene in murine models leading to decreased tumour size and vascularisation [75-77]. Unsurprisingly, the role of $\alpha_{2} \beta_{1}$ integrin in mediating angiogenesis is dynamic and involves crosstalk between different integrins and the cellular microenvironment. A number of inhibitory antibodies, bioactive molecules, and small protein fragments have been developed that aim to prevent angiogenesis by eliminating $\alpha_{2} \beta_{1}$ expression. Early attempts to abrogate angiogenesis via the VEGF pathway and $\alpha_{2}$ integrin utilised anti- $\alpha_{2}$ monoclonal antibodies. Nude mice with human squamous cell carcinoma xenografts displayed a $40 \%$ decrease in tumour growth and a reduction in angiogenesis by $60 \%$ when treated with an anti- $\alpha_{2}$ antibody [78]. Furthermore, the anti- $\alpha_{2}$ antibody HA1/29 reduced endothelial cell migration in an immobilized collagen type 1 assay by approximately $40 \%$ [78]. It has been suggested that these anti- $\alpha_{2}$ antibodies could be used as human angiogenesis inhibitors in tumour therapy [9].

6.1. Disintegrins and $\alpha_{2} \beta_{1}$. Small peptide agonists of integrins derived from protein fragments and snake venom are known to bind to $\alpha_{2} \beta_{1}$ in particular, thus presenting an exciting avenue for therapeutic intervention. These disintegrins usually contain the RGD motif, although this is not always the case [79]. Disintegrins such as angiocidin, a thrombospondin-1 binding protein, have been demonstrated to have antitumour effects by inhibiting angiogenesis. Importantly, angiocidin binds to $\alpha_{2} \beta_{1}$ and type I collagen with high affinity [80] and has been shown to bind to prostate, breast, melanoma, and colon cancer cell lines expressing $\alpha_{2} \beta_{1}$. Furthermore, expression levels of angiocidin in human colon cancer tissues have been shown to correlate with disease burden [80].

Similarly, snake venom derived disintegrins such as rhodocytin and jararhagin prevent angiogenesis. Both rhodocytin and jararhagin have a high affinity for $\alpha_{2} \beta_{1}$, and the former has been demonstrated to completely block the adhesion of fibrosarcoma cells to type 1 collagen preventing signalling [81], perhaps decreasing the amount of crosstalk between integrins. These molecules have been shown to have antitumour activity in in vitro and in vivo models; however, both are yet to be tested in prostate cancer models. Importantly, both rhodocytin and jararhagin are soluble, have small-molecular-weights, and by nature are able to move efficiently through tissue and the ECM [81]. Furthermore, E7820 is a small molecular weight inhibitor of integrin $\alpha_{2} \beta_{1}$ that has reached Phase II of human trial [82]. An aromatic sulphonamide derivative, E7820, has been shown to inhibit tubule formation in human umbilical vascular endothelial cells (HUVEC) by binding to, and inhibiting the expression of, the $\alpha_{2}$ subunit [83]. Phase II human trials are currently underway, which aim to examine the combinatory effect of E7820 with the traditional chemotherapeutic agent FOLFIRI (FOL-folinic acid, F-5-fluorouracil, and IRI-Irinotecan), and also E7820 with the monoclonal antibody cetuximab (Erbitux), in patients with metastatic colorectal cancer. In addition, the utilisation of $\alpha_{2}$ expression as a biomarker for E7820 efficacy is currently underway $[84,85]$. The results of this study indicated that a relatively low knockdown of 
$\alpha_{2}$ expression achieved tumour stasis at biologically apposite levels for human administration [84].

\section{Conclusion}

The integrins $\alpha_{2}$ and $\alpha_{6}$ have emerged as putative biological markers for evaluation of prostate cancer susceptibility and also progression and metastasis. The combination of cell surface receptor coupled with differential expression is particularly important for the elucidation of the mechanisms that drive prostate cancer pathogenesis. The identification of the genetic drivers of tumour development, through use of new high-throughput genetic technologies combined with our knowledge of the biology of prostate cancer, is opening the door for the generation of new patient tailored therapies. However, whilst more than 40 disease susceptibility loci have been identified, the majority of loci identified confer only a modest risk and collectively account for approximately $30 \%$ of heritable disease burden; thus, further elucidation of the underlying genetic contributors is required. Inclusion of both common and rare prostate cancer variants in polygenic risk calculators is likely to improve their predictive value in diagnosis and prognosis.

Differential expression of the $\alpha_{2}$ integrins in prostate tumour cells has made them useful targets for both imaging techniques and therapeutics. Radiolabelled peptides, which ligate integrin $\alpha_{2} \beta_{1}$, have recently been developed for PET scanning with great success, allowing precise imaging of prostate tumour cells to occur in real time. The correlation with $\alpha_{2} \beta_{1}$ expression and prostate cancer has been a primary target in therapeutics. By targeting $\alpha_{2} \beta_{1}$, angiogenesis can be inhibited by preventing ligation with type 1 collagen, which is required for the formation of new blood vessels. Furthermore, the expression of $\alpha_{2}$ on platelets has been successfully utilised as a biomarker to examine the efficacy of an agonist targeting $\alpha_{2}$ in tumour cells in phase I and II human trials. Thus the existing knowledge of integrin biology and in particular the identification of $\alpha_{2}$ and $\alpha_{6}$, as key drivers of prostate cancer pathogenesis, is facilitating translation into new diagnostic and therapeutic applications. Further work is required to gain a thorough understanding of the genetic drivers identified for prostate cancer and how they influence tumour biology. This will permit informed application of our genetic discoveries in the clinical setting.

\section{Acknowledgments}

The authors would like to thank Dr. Rebekah McWhirter and Dr. Adele Holloway for assistance with revisions of this paper. The authors would also like to thank the funding bodies supporting the Tasmanian Inherited Cancer Centre including the Australian Cancer Research Foundation, Cancer Australia, Cancer Council Tasmania, Leukaemia Foundation, David Collins Leukaemia Foundation, and the Royal Hobart Hospital Research Foundation.

\section{References}

[1] M. Provenzano, "New biomarkers in prostate cancer," Praxis (Bern. 1994), vol. 101, no. 2, pp. 115-121, 2012.

[2] J. Ivaska and J. Heino, "Adhesion receptors and cell invasion: mechanisms of integrin-guided degradation of extracellular matrix," Cellular and Molecular Life Sciences, vol. 57, no. 1, pp. 16-24, 2000.

[3] X. Lu, D. Lu, M. Scully, and V. Kakkar, "The role of integrins in cancer and the development of anti-integrin therapeutic agents for cancer therapy," Perspectives in Medicinal Chemistry, vol. 2008, no. 2, pp. 57-73, 2008.

[4] M. Shimaoka, J. Takagi, and T. A. Springer, "Conformational regulation of integrin structure and function," Annu Rev Biophys Biomol Struct, vol. 31, pp. 485-516, 2002.

[5] R. D. Burke, "Invertebrate integrins: structure, function, and evolution," International Review of Cytology, vol. 191, pp. 257284, 1999.

[6] H. L. Goel, N. Alam, I. N. S. Johnson, and L. R. Languino, "Integrin signaling aberrations in prostate cancer," American Journal of Translational Research, vol. 1, no. 3, pp. 211-220, 2009.

[7] F. G. Giancotti and E. Ruoslahti, "Integrin signaling," Science, vol. 285, no. 5430, pp. 1028-1032, 1999.

[8] G. J. Thomas, S. Poomsawat, M. P. Lewis, I. R. Hart, P. M. Speight, and J. F. Marshall, "Alpha v beta 6 Integrin upregulates matrix metalloproteinase 9 and promotes migration of normal oral keratinocytes," Journal of Investigative Dermatology, vol. 116, no. 6, pp. 898-904, 2001.

[9] G. Alghisi and C. Rüegg, "Vascular integrins in tumor angiogenesis: mediators and therapeutic targets," Endothelium, vol. 13, no. 2, pp. 113-135, 2006.

[10] C. J. Avraamides, B. Garmy-Susini, and J. A. Varner, "Integrins in angiogenesis and lymphangiogenesis," Nature Reviews Cancer, vol. 8, no. 8, pp. 604-617, 2008.

[11] B. Wehrle-Haller and B. A. Imhof, "Integrin-dependent pathologies," Journal of Pathology, vol. 200, no. 4, pp. 481-487, 2003.

[12] N. E. Ramirez, Z. Zhang, A. Madamanchi et al., "The $\alpha 2 \beta 1$ integrin is a metastasis suppressor in mouse models and human cancer," Journal of Clinical Investigation, vol. 121, no. 1, pp. 226-237, 2011.

[13] P. S. Steeg, "Tumor metastasis: mechanistic insights and clinical challenges," Nature Medicine, vol. 12, no. 8, pp. 895904, 2006.

[14] J. S. Desgrosellier and D. A. Cheresh, "Integrins in cancer: biological implications and therapeutic opportunities," Nature Reviews Cancer, vol. 10, no. 1, pp. 9-22, 2010.

[15] M. Millard, S. Odde, and N. Neamati, "Integrin targetted theapeutics," Theranostics, vol. 1, pp. 154-188, 2011.

[16] L. Bubendorf, A. Schöpfer, U. Wagner et al., "Metastatic patterns of prostate cancer: an autopsy study of 1,589 patients," Human Pathology, vol. 31, no. 5, pp. 578-583, 2000.

[17] T. Riikonen, J. Westermarck, L. Koivisto, A. Broberg, V. M. Kahari, and J. Heino, "Integrin $\alpha 2 \beta 1$ is a positive regulator of collagenase (MMP-1) and collagen $\alpha 1$ (I) gene expression," Journal of Biological Chemistry, vol. 270, no. 22, pp. 1354813552, 1995.

[18] P. J. Kostenuik, O. Sanchez-Sweatman, F. William Orr, and G. Singh, "Bone cell matrix promotes the adhesion of human prostatic carcinoma cells via the $\alpha 2 \beta 1$ integrin," Clinical and Experimental Metastasis, vol. 14, no. 1, pp. 19-26, 1996.

[19] S. H. Lang, N. W. Clarke, N. J. R. George, and N. G. Testa, "Primary prostatic epithelial cell binding to human bone 
marrow stroma and the role of $\alpha 2 \beta 1$ integrin," Clinical and Experimental Metastasis, vol. 15, no. 3, pp. 218-227, 1997.

[20] C. L. Hall, J. Dai, K. L. Van Golen, E. T. Keller, and M. W. Long, "Type I collagen receptor $(\alpha 2 \beta 1)$ signaling promotes the growth of human prostate cancer cells within the bone," Cancer Research, vol. 66, no. 17, pp. 8648-8654, 2006.

[21] H. Bonkhoff, U. Stein, and K. Remberger, "Differential expression of $\alpha 6$ and $\alpha 2$ very late antigen integrins in the normal, hyperplastic, and neoplastic prostate: simultaneous demonstration of cell surface receptors and their extracellular ligands," Human Pathology, vol. 24, no. 3, pp. 243-248, 1993.

[22] B. S. Knudsen and C. K. Miranti, "The impact of cell adhesion changes on proliferation and survival during prostate cancer development and progression," Journal of Cellular Biochemistry, vol. 99, no. 2, pp. 345-361, 2006.

[23] J. Pontes-Junior, S. T. Reis, M. Dall'oglio et al., "Evaluation of the expression of integrins and cell adhesion molecules through tissue microarray in lymph node metastases of prostate cancer," Journal of Carcinogenesis, vol. 8, no. 1, p. 3, 2009.

[24] R. Edgar, M. Domrachev, and A. E. Lash, "Gene expression omnibus: NCBI gene expression and hybridization array data repository," Nucleic Acids Research, vol. 30, no. 1, pp. 207-210, 2002.

[25] J. D. Knox, A. E. Cress, V. Clark et al., "Differential expression of extracellular matrix molecules and the $\alpha 6$ - integrins in the normal and neoplastic prostate," American Journal of Pathology, vol. 145, no. 1, pp. 167-174, 1994.

[26] T. Mirtti, C. Nylund, J. Lehtonen et al., "Regulation of prostate cell collagen receptors by malignant transformation," International Journal of Cancer, vol. 118, no. 4, pp. 889-898, 2006.

[27] A. T. Collins and N. J. Maitland, "Prostate cancer stem cells," European Journal of Cancer, vol. 42, no. 9, pp. 1213-1218, 2006.

[28] H. Li, X. Chen, T. Calhoun-Davis, K. Claypool, and D. G. Tang, "PC3 human prostate carcinoma cell holoclones contain selfrenewing tumor-initiating cells," Cancer Research, vol. 68, no. 6, pp. 1820-1825, 2008.

[29] G. J. Klarmann, E. M. Hurt, L. A. Mathews et al., "Invasive prostate cancer cells are tumor initiating cells that have a stem cell-like genomic signature," Clinical and Experimental Metastasis, vol. 26, no. 5, pp. 433-446, 2009.

[30] N. Guzmán-Ramírez, M. Völler, A. Wetterwald et al., "In vitro propagation and characterization of neoplastic stem/ progenitor-like cells from human prostate cancer tissue," Prostate, vol. 69, no. 15, pp. 1683-1693, 2009.

[31] A. T. Collins, P. A. Berry, C. Hyde, M. J. Stower, and N. J. Maitland, "Prospective identification of tumorigenic prostate cancer stem cells," Cancer Research, vol. 65, no. 23, pp. 1094610951, 2005.

[32] J. Miki and J. S. Rhim, "Prostate cell cultures as in vitro models for the study of normal stem cells and cancer stem cells," Prostate Cancer and Prostatic Diseases, vol. 11, no. 1, pp. 3239, 2008.

[33] C. L. Goh, F. R. Schumacher, D. Easton et al., "Genetic variants associated with predisposition to prostate cancer and potential clinical implications," Journal of Internal Medicine, vol. 271, no. 4, pp. 353-365, 2012.

[34] S. Tao, Z. Wang, J. Feng et al., "A genome-wide search for loci interacting with known prostate cancer risk-associated genetic variants," Carcinogenesis, vol. 33, no. 3, pp. 598-603, 2012.
[35] T. A. Manolio, "Genomewide association studies and assessment of the risk of disease," New England Journal of Medicine, vol. 363, no. 2, pp. 166-176, 2010.

[36] M. Aly, F. Wiklund, J. Xu et al., "Polygenic risk score improves prostate cancer risk prediction: results from the Stockholm1 cohort study," European Urology, vol. 60, no. 1, pp. 21-28, 2011.

[37] S. L. Zheng, J. Sun, F. Wiklund et al., "Cumulative association of five genetic variants with prostate cancer," New England Journal of Medicine, vol. 358, no. 9, pp. 910-919, 2008.

[38] N. Pashayan, S. W. Duffy, S. Chowdhury et al., "Polygenic susceptibility to prostate and breast cancer: implications for personalised screening," British Journal of Cancer, vol. 104, no. 10, pp. 1656-1663, 2011.

[39] S. M. Edwards, D. G. R. Evans, Q. Hope et al., "Prostate cancer in BRCA2 germline mutation carriers is associated with poorer prognosis," British Journal of Cancer, vol. 103, no. 6, pp. 918-924, 2010.

[40] S. M. Edwards, Z. Kote-Jarai, J. Meitz et al., "Two percent of men with early-onset prostate cancer harbor germline mutations in the BRCA2 gene," American Journal of Human Genetics, vol. 72, no. 1, pp. 1-12, 2003.

[41] E. T. Cirulli and D. B. Goldstein, "Uncovering the roles of rare variants in common disease through whole-genome sequencing," Nature Reviews Genetics, vol. 11, no. 6, pp. 415425,2010

[42] S. V. Ramagopalan, D. A. Dyment, M. Z. Cader et al., "Rare variants in the CYP27B1 gene are associated with multiple sclerosis," Annals of Neurology, vol. 70, no. 6, pp. 881-886, 2011.

[43] C. M. Ewing, A. M. Ray, E. M. Lange et al., "Germline mutations in HOXB13 and prostate-cancer risk," New England Journal of Medicine, vol. 366, no. 2, pp. 141-149, 2012.

[44] M. Deb, D. Sengupta, and S. K. Patra, "Integrin-epigenetics: a system with imperative impact on cancer," Cancer Metastasis, vol. 31, no. 1-2, pp. 221-234, 2012.

[45] R. Maruyama, S. Toyooka, K. O. Toyooka et al., "Aberrant promoter methylation profile of prostate cancers and its relationship to clinicopathological features," Clinical Cancer Research, vol. 8, no. 2, pp. 514-519, 2002.

[46] C. Jerónimo, P. J. Bastian, A. Bjartell et al., "Epigenetics in prostate cancer: biologic and clinical relevance," European Urology, vol. 60, no. 4, pp. 753-766, 2011.

[47] C. Albany, A. S. Alva, A. M. Aparicio et al., "Epigenetics in prostate cancer," Prostate Cancer, vol. 2011, Article ID 580318, 12 pages, 2011.

[48] M. T. Bedford and P. D. Van Helden, "Hypomethylation of DNA in pathological conditions of the human prostate," Cancer Research, vol. 47, no. 20, pp. 5274-5276, 1987.

[49] W. G. Nelson, S. Yegnasubramanian, A. T. Agoston et al., "Abnormal DNA methylation, epigenetics, and prostate cancer," Frontiers in Bioscience, vol. 12, no. 11, pp. 4254-4266, 2007.

[50] J. H. Kim, S. M. Dhanasekaran, J. R. Prensner et al., "Deep sequencing reveals distinct patterns of DNA methylation in prostate cancer," Genome Research, vol. 21, no. 7, pp. 10281041, 2011.

[51] L. Van Neste, J. G. Herman, G. Otto, J. W. Bigley, J. I. Epstein, and W. Van Criekinge, "The Epigenetic promise for prostate cancer diagnosis," Prostate, vol. 72, no. 11, pp. 1248-1261, 2012.

[52] O. Cussenot, A. R. Azzouzi, G. Bantsimba-Malanda et al., "Effect of genetic variability within 8q24 on aggressiveness 
patterns at diagnosis and familial status of prostate cancer," Clinical Cancer Research, vol. 14, no. 17, pp. 5635-5639, 2008.

[53] B. T. Helfand, S. Loeb, J. Cashy et al., "Tumor Characteristics of Carriers and Noncarriers of the deCODE 8q24 Prostate Cancer Susceptibility Alleles," Journal of Urology, vol. 179, no. 6, pp. 2197-2202, 2008.

[54] L. M. FitzGerald, E. M. Kwon, M. P. Conomos et al., "Genome-wide association study identifies a genetic variant associated with risk for more aggressive prostate cancer," Cancer Epidemiology Biomarkers and Prevention, vol. 20, no. 6, pp. 1196-1203, 2011.

[55] I. P. Gorlov, G. E. Gallick, O. Y. Gorlova, C. Amos, and C. J. Logothetis, "GWAS meets microarray: are the results of genome-wide association studies and gene-expression profiling consistent? Prostate cancer as an example," PLoS ONE, vol. 4, no. 8, Article ID e6511, 2009.

[56] R. A. Eeles, Z. Kote-Jarai, A. A. Al Olama et al., "Identification of seven new prostate cancer susceptibility loci through a genome-wide association study," Nature Genetics, vol. 41, no. 10, pp. 1116-1121, 2009.

[57] I. Cheng, S. J. Plummer, C. Neslund-Dudas et al., "Prostate cancer susceptibility variants confer increased risk of disease progression," Cancer Epidemiology Biomarkers and Prevention, vol. 19, no. 9, pp. 2124-2132, 2010.

[58] L. M. FitzGerald, B. Patterson, R. Thomson et al., "Identification of a prostate cancer susceptibility gene on chromosome $5 \mathrm{p} 13 \mathrm{q} 12$ associated with risk of both familial and sporadic disease," European Journal of Human Genetics, vol. 17, no. 3, pp. 368-377, 2009.

[59] U. Langsenlehner, W. Renner, B. Yazdani-Biuki et al., "Integrin alpha-2 and beta-3 gene polymorphisms and breast cancer risk," Breast Cancer Research and Treatment, vol. 97, no. 1, pp. 67-72, 2006.

[60] E. Vairaktaris, C. Yapijakis, S. Derka et al., "Association of platelet glycoprotein Ia polymorphism with minor increase of risk for oral cancer," European Journal of Surgical Oncology, vol. 32, no. 4, pp. 455-457, 2006.

[61] C. Kimchi-Sarfaty, J. M. Oh, I. W. Kim et al., "A 'silent' polymorphism in the MDR1 gene changes substrate specificity," Science, vol. 315, no. 5811, pp. 525-528, 2007.

[62] A. Katsnelson, "Breaking the silence," Nature Medicine, vol. 17, no. 12, pp. 1536-1538, 2011.

[63] B. Jacquelin, M. D. Tarantino, M. Kritzik et al., "Alleledependent transcriptional regulation of the human integrin $\alpha 2$ gene," Blood, vol. 97, no. 6, pp. 1721-1726, 2001.

[64] Y. Ye, B. Xu, G. V. Nikiforovich, S. Bloch, and S. Achilefu, "Exploring new near-infrared fluorescent disulfide-based cyclic RGD peptide analogs for potential integrin-targeted optical imaging," Bioorganic and Medicinal Chemistry Letters, vol. 21, no. 7, pp. 2116-2120, 2011.

[65] C. W. Huang, Z. Li, H. Cai, T. Shahinian, and P. S. Conti, "Novel $\alpha 2 \beta 1$ integrin-targeted peptide probes for prostate cancer imaging," Molecular Imaging, vol. 10, no. 4, pp. 284294, 2011.

[66] C. W. Huang, Z. Li, H. Cai, K. Chen, T. Shahinian, and P. S. Conti, "Design, synthesis and validation of integrin $\alpha 2 \beta$ 1-targeted probe for microPET imaging of prostate cancer," European Journal of Nuclear Medicine and Molecular Imaging, vol. 38, no. 7, pp. 1313-1322, 2011.

[67] C. W. Huang, Z. Li, and P. S. Conti, "In vivo near-infrared fluorescence imaging of integrin alpha2betal in prostate cancer with cell-penetrating-peptide-conjugated DGEA probe,"
Journal of Nuclear Medicine, vol. 52, no. 12, pp. 1979-1986, 2011.

[68] E. Giovannucci, "A review of epidemiologic studies of tomatoes, lycopene, and prostate cancer," Experimental Biology and Medicine, vol. 227, no. 10, pp. 852-859, 2002.

[69] V. A. Kirsh, S. T. Mayne, U. Peters et al., "A prospective study of lycopene and tomato product intake and risk of prostate cancer," Cancer Epidemiology Biomarkers and Prevention, vol. 15, no. 1, pp. 92-98, 2006.

[70] D. Ilic, K. M. Forbes, and C. Hassed, "Lycopene for the prevention of prostate cancer," Cochrane database of systematic reviews, vol. 11, Article ID 008007, 2011.

[71] D. Heber and Q. Y. Lu, "Overview of mechanisms of action of lycopene," Experimental Biology and Medicine, vol. 227, no. 10, pp. 920-923, 2002.

[72] T. Bureyko, H. Hurdle, J. B. Metcalfe, M. T. Clandinin, and V. C. Mazurak, "Reduced growth and integrin expression of prostate cells cultured with lycopene, vitamin E and fish oil in vitro," British Journal of Nutrition, vol. 101, no. 7, pp. 990-997, 2009.

[73] M. J. Magbanua, R. Roy, E. V. Sosa et al., "Gene expression and biological pathways in tissue of men with prostate cancer in a randomized clinical trial of lycopene and fish oil supplementation," PLoS One, vol. 6, no. 9, Article ID e24004, 2011.

[74] Z. Gavish, J. H. Pinthus, V. Barak et al., "Growth inhibition of cancer xenografts by halofuginone," prostate, vol. 51, no. 2, pp. 73-83, 2002.

[75] Z. Zhang, N. E. Ramirez, T. E. Yankeelov et al., “ $\alpha 2 \beta 1$ integrin expression in the tumor microenvironment enhances tumor angiogenesis in a tumor cell-specific manner," Blood, vol. 111, no. 4, pp. 1980-1988, 2008.

[76] A. Pozzi, W. F. LeVine, and H. A. Gardner, "Low plasma levels of matrix metalloproteinase 9 permit increased tumor angiogenesis," Oncogene, vol. 21, no. 2, pp. 272-281, 2002.

[77] A. Pozzi, P. E. Moberg, L. A. Miles, S. Wagner, P. Soloway, and H. A. Gardner, "Elevated matrix metalloprotease and angiostatin levels in integrin $\alpha 1$ knockout mice cause reduced tumor vascularization," Proceedings of the National Academy of Sciences of the United States of America, vol. 97, no. 5, pp. 2202-2207, 2000.

[78] D. R. Senger, C. A. Perruzzi, M. Streit, V. E. Koteliansky, A. R. De Fougerolles, and M. Detmar, "The $\alpha 1 \beta 1$ and $\alpha 2 \beta 1$ integrins provide critical support for vascular endothelial growth factor signaling, endothelial cell migration, and tumor angiogenesis," American Journal of Pathology, vol. 160, no. 1, pp. 195-204, 2002.

[79] J. A. Eble, S. Niland, A. Dennes, A. Schmidt-Hederich, P. Bruckner, and G. Brunner, "Rhodocetin antagonizes stromal tumor invasion in vitro and other $\alpha 2 \beta 1$ integrin-mediated cell functions," Matrix Biology, vol. 21, no. 7, pp. 547-558, 2002.

[80] Y. Sabherwal, V. L. Rothman, S. Dimitrov et al., "Integrin $\alpha 2 \beta 1$ mediates the anti-angiogenic and anti-tumor activities of angiocidin, a novel tumor-associated protein," Experimental Cell Research, vol. 312, no. 13, pp. 2443-2453, 2006.

[81] J. A. Eble, "Collagen-binding integrins as pharmaceutical targets," Current Pharmaceutical Design, vol. 11, no. 7, pp. 867-880, 2005.

[82] Irinotecan Plus E7820 Versus FOLFIRI in Second-Line Therapy in Patients With Locally Advanced or Metastatic Colon or Rectal Cancer, http://clinicaltrials.gov/ct2/show/record/NCT01347645? recr=Open\&cond $=$ colon + cancer\&lup_s $=$ 06\%2F04\%2F2010\&lup_d=360. 
[83] Y. Funahashi, N. H. Sugi, T. Semba et al., "Sulfonamide derivative, E7820, is a unique angiogenesis inhibitor suppressing an expression of integrin $\alpha 2$ subunit on endothelium," Cancer Research, vol. 62, no. 21, pp. 6116-6123, 2002.

[84] R. J. Keizer, Y. Funahashi, T. Semba et al., "Evaluation of $\alpha 2$-integrin expression as a biomarker for tumor growth inhibition for the investigational integrin inhibitor E7820 in preclinical and clinical studies," AAPS Journal, vol. 13, no. 2, pp. 230-239, 2011.

[85] R. J. Keizer, M. K. Zamacona, M. Jansen et al., "Application of population pharmacokinetic modeling in early clinical development of the anticancer agent E7820," Investigational New Drugs, vol. 27, no. 2, pp. 140-152, 2009. 


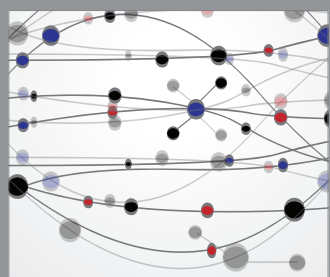

The Scientific World Journal
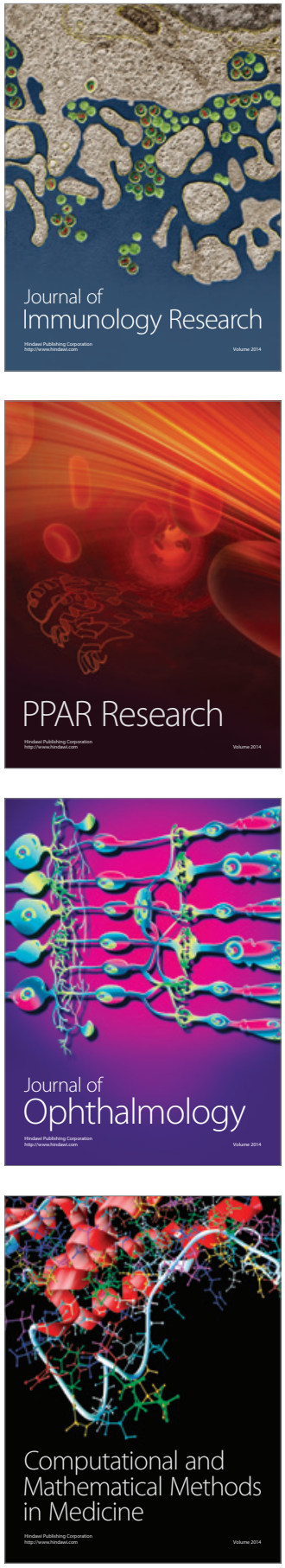

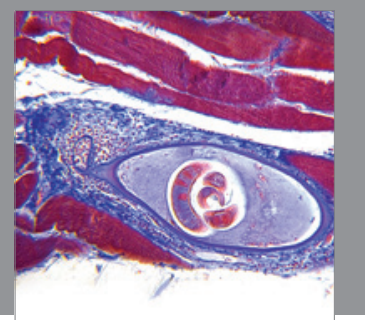

Gastroenterology

Research and Practice
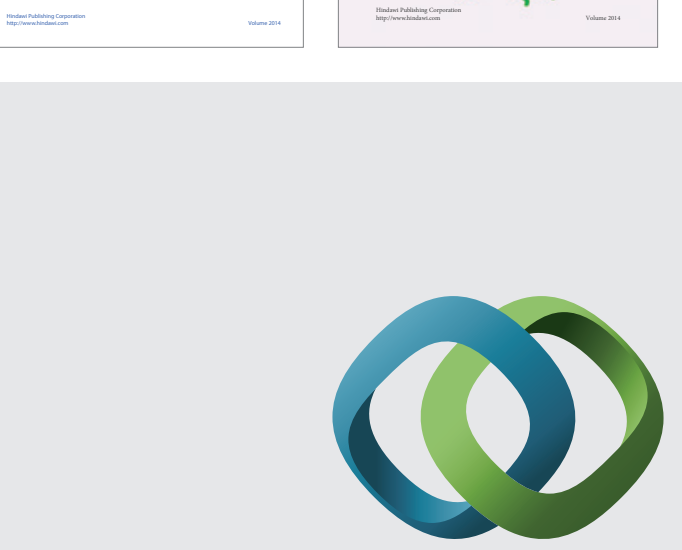

\section{Hindawi}

Submit your manuscripts at

http://www.hindawi.com
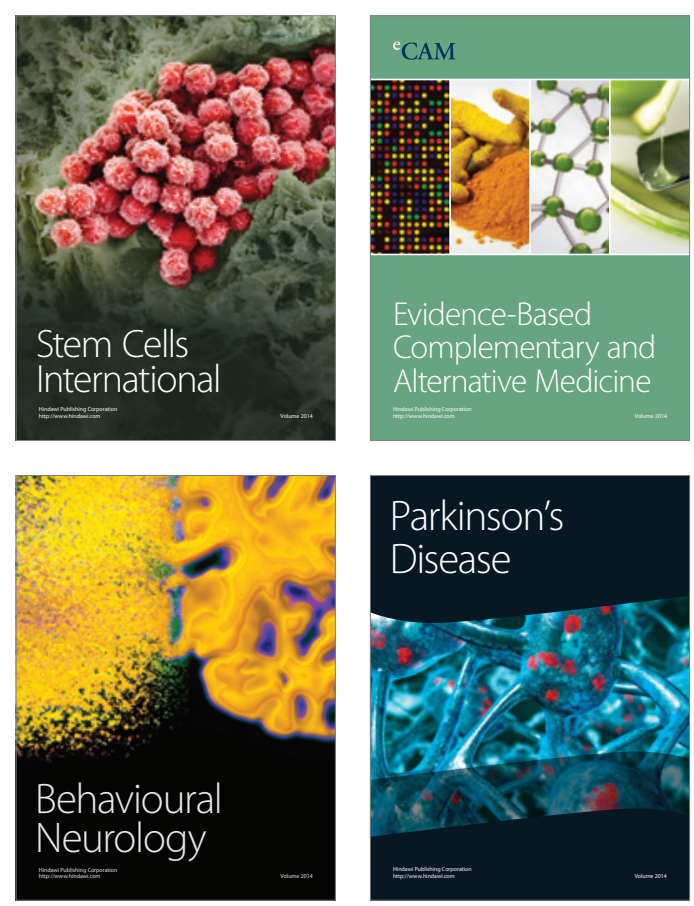

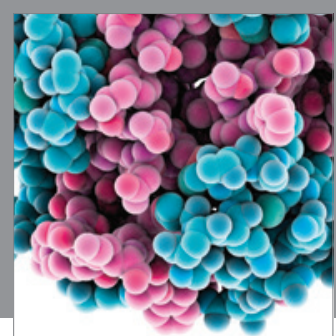

Journal of
Diabetes Research

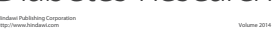

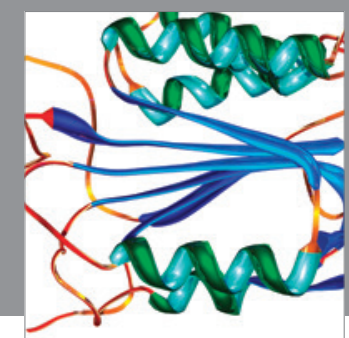

Disease Markers
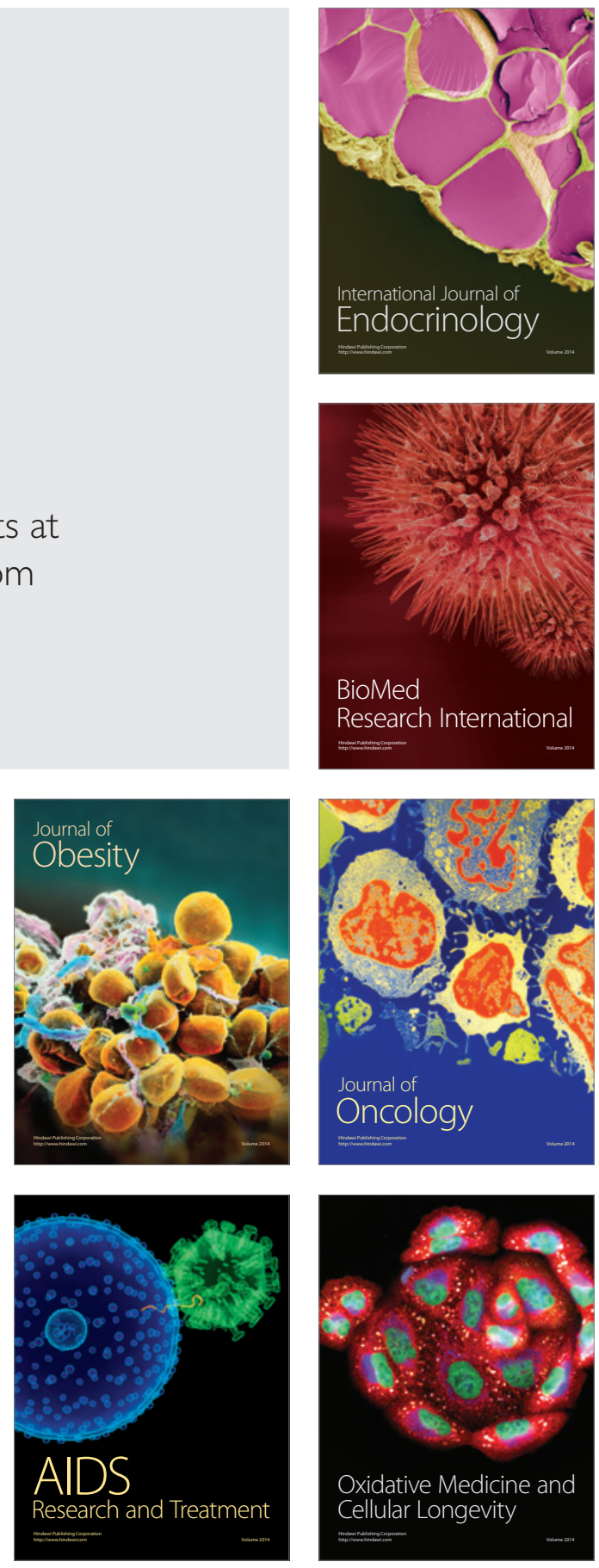